\title{
BSREM reconstruction for improved detection of in-transit metastases with digital FDG-PET/CT in patients with malignant melanoma.
}

\author{
Aljared, Arwa ; Alharbi, Abdullah Awad ; Huellner, Martin W
}

\begin{abstract}
Block sequential regularized expectation maximization (BSREM) is a Bayesian penalizedlikelihood reconstruction algorithm for PET, which reaches full convergence without the detriment of deteriorating the image quality by noise. Therefore, BSREM might have implications particularly for the detection of small lesions, which may be beneficial in melanoma patients. Our case of a 70-yearold man with metastasized malignant melanoma illustrates the impact of such a novel iterative PET reconstruction algorithm. Whereas the lymph node metastases are seen with the latest generation ordered subset expectation maximization reconstruction, the in-transit metastases are identified straightforward only with BSREM reconstruction.
\end{abstract}

DOI: https://doi.org/10.1097/RLU.0000000000002024

Posted at the Zurich Open Repository and Archive, University of Zurich ZORA URL: https://doi.org/10.5167/uzh-152842

Journal Article

Published Version

Originally published at:

Aljared, Arwa; Alharbi, Abdullah Awad; Huellner, Martin W (2018). BSREM reconstruction for improved detection of in-transit metastases with digital FDG-PET/CT in patients with malignant melanoma. Clinical Nuclear Medicine, 43(5):370-371.

DOI: https://doi.org/10.1097/RLU.0000000000002024 


\title{
BSREM Reconstruction for Improved Detection of In-Transit Metastases With Digital FDG-PET/CT in Patients With Malignant Melanoma
}

\author{
Arwa Aljared, MD, Abdullah Awad Alharbi, MD, and Martin W. Huellner, MD
}

\begin{abstract}
Block sequential regularized expectation maximization (BSREM) is a Bayesian penalized-likelihood reconstruction algorithm for PET, which reaches full convergence without the detriment of deteriorating the image quality by noise. Therefore, BSREM might have implications particularly for the detection of small lesions, which may be beneficial in melanoma patients. Our case of a 70-year-old man with metastasized malignant melanoma illustrates the impact of such a novel iterative PET reconstruction algorithm. Whereas the lymph node metastases are seen with the latest generation ordered subset expectation maximization reconstruction, the in-transit metastases are identified straightforward only with BSREM reconstruction.
\end{abstract}

Key Words: BSREM, malignant melanoma, in-transit metastasis, FDG, PET/CT, OSEM, PSF

(Clin Nucl Med 2018;43: 370-371)

Received for publication December 22, 2017; revision accepted January 28, 2018 From the Department of Nuclear Medicine, University Hospital Zurich/University of Zurich, Zurich, Switzerland.

Conflicts of interest and sources of funding: none declared.

Correspondence to: Arwa Aljared, MD, Department of Nuclear Medicine, University Hospital Zurich/University of Zurich, Rämistrasse 100, 8091 Zurich, Switzerland. E-mail: Dr.arwa@gmail.com.

Copyright (C) 2018 Wolters Kluwer Health, Inc. All rights reserved.

ISSN: 0363-9762/18/4305-0370

DOI: $10.1097 /$ RLU.0000000000002024

\section{REFERENCES}

1. Read RL, Haydu L, Saw RP, et al. In-transit melanoma metastases: incidence, prognosis, and the role of lymphadenectomy. Ann Surg Oncol. 2015; $22: 475-481$

2. Singnurkar A, Wang J, Joshua AM, et al. ${ }^{18}$ F-FDG-PET/CT in the staging and management of melanoma: a prospective multicenter Ontario PET registry study. Clin Nucl Med. 2016;41:189-193.

3. Covington MF, Curiel CN, Lattimore L, et al. FDG-PET/CT for monitoring response of melanoma to the novel oncolytic viral therapy talimogene laherparepvec. Clin Nucl Med. 2017;42:114-115.

4. Mena E, Taghipour M, Sheikhbahaei S, et al. ${ }^{18}$ F-FDG-PET/CT in the staging and management of melanoma: a prospective multicenter Ontario PET registry study. Clin Nucl Med. 2016;41:e403-409.

5. Drouet C, Morel O, Boulahdour H. Bilateral huge incidentalomas of isolated adrenal metastases from unknown primary melanoma revealed by ${ }^{18} \mathrm{~F}-\mathrm{FDG}$ PET/CT. Clin Nucl Med. 2017;42:e51-e53.

6. Baratto L, Park SY, Hatami N, et al. ${ }^{18}$ F-FDG silicon photomultiplier PET/ CT: a pilot study comparing semi-quantitative measurements with standard PET/CT. PLoS One. 2017;12:e0178936.

7. Sah BR, Stolzmann P, Delso G, et al. Clinical evaluation of a block sequential regularized expectation maximization reconstruction algorithm in ${ }^{18}$ F-FDG PET/CT studies. Nucl Med Commun. 2017;38:57-66.

8. Enilorac B, Lasnon C, Nganoa C, et al. Does PET reconstruction method affect Deauville scoring in lymphoma patients? J Nucl Med. 2017. [Epub ahead of print].

9. Chilcott AK, Bradley KM, McGowan DR. Effect of a Bayesian penalized likelihood PET reconstruction compared with ordered subset expectation maximization on clinical image quality over a wide range of patient weights AJR Am J Roentgenol. 2018;210:153-157.

10. Parvizi N, Franklin JM, McGowan DR, et al. Does a novel penalized likelihood reconstruction of ${ }^{18}$ F-FDG PET-CT improve signal-to-background in colorectal liver metastases? Eur J Radiol. 2015;84:1873-1878.

11. Sampaio Vieira T, Borges Faria D, Azevedo Silva F, et al. The impact of a Bayesian penalized likelihood reconstruction algorithm on the evaluation of indeterminate pulmonary nodules by dual-time point ${ }^{18} \mathrm{~F}$-FDG PET/CT. Clin Nucl Med. 2017;42:e352-e354.

12. Akamatsu G, Ishikawa $\mathrm{K}$, Mitsumoto $\mathrm{K}$, et al. Improvement in PET/CT image quality with a combination of point-spread function and time-of-flight in relation to reconstruction parameters. J Nucl Med. 2012;53:1716-1722.

13. van der Vos CS, Koopman D, Rijnsdorp S, et al. Quantification, improvement, and harmonization of small lesion detection with state-of-the-art PET. Eur J Nucl Med Mol Imaging. 2017;44:4-16.

14. Nuyts J, Fessler JA. A penalized-likelihood image reconstruction method for emission tomography, compared to postsmoothed maximum-likelihood with matched spatial resolution. IEEE Trans Med Imaging. 2003;22:1042-1052.

15. Teoh EJ, McGowan DR, Macpherson RE, et al. Phantom and clinical evaluation of the Bayesian penalized likelihood reconstruction algorithm Q.Clear on an LYSO PET/CT system. J Nucl Med. 2015;56:1447-1452.

16. Ahn S, Ross SG, Asma E, et al. Quantitative comparison of OSEM and penalized likelihood image reconstruction using relative difference penalties for clinical PET. Phys Med Biol. 2015;60:5733-5751.

17. Reynes-Llompart G, Gamez-Cenzano C, Romero-Zayas I, et al. Performance characteristics of the whole-body discovery IQ PET/CT system. $J$ Nucl Med. 2017;58:1155-1161.

18. Vallot D, Caselles O, Chaltiel L, et al. A clinical evaluation of the impact of the Bayesian penalized likelihood reconstruction algorithm on PET FDG metrics. Nucl Med Commun. 2017;38:979-984. 


\section{OSEM + PSF}

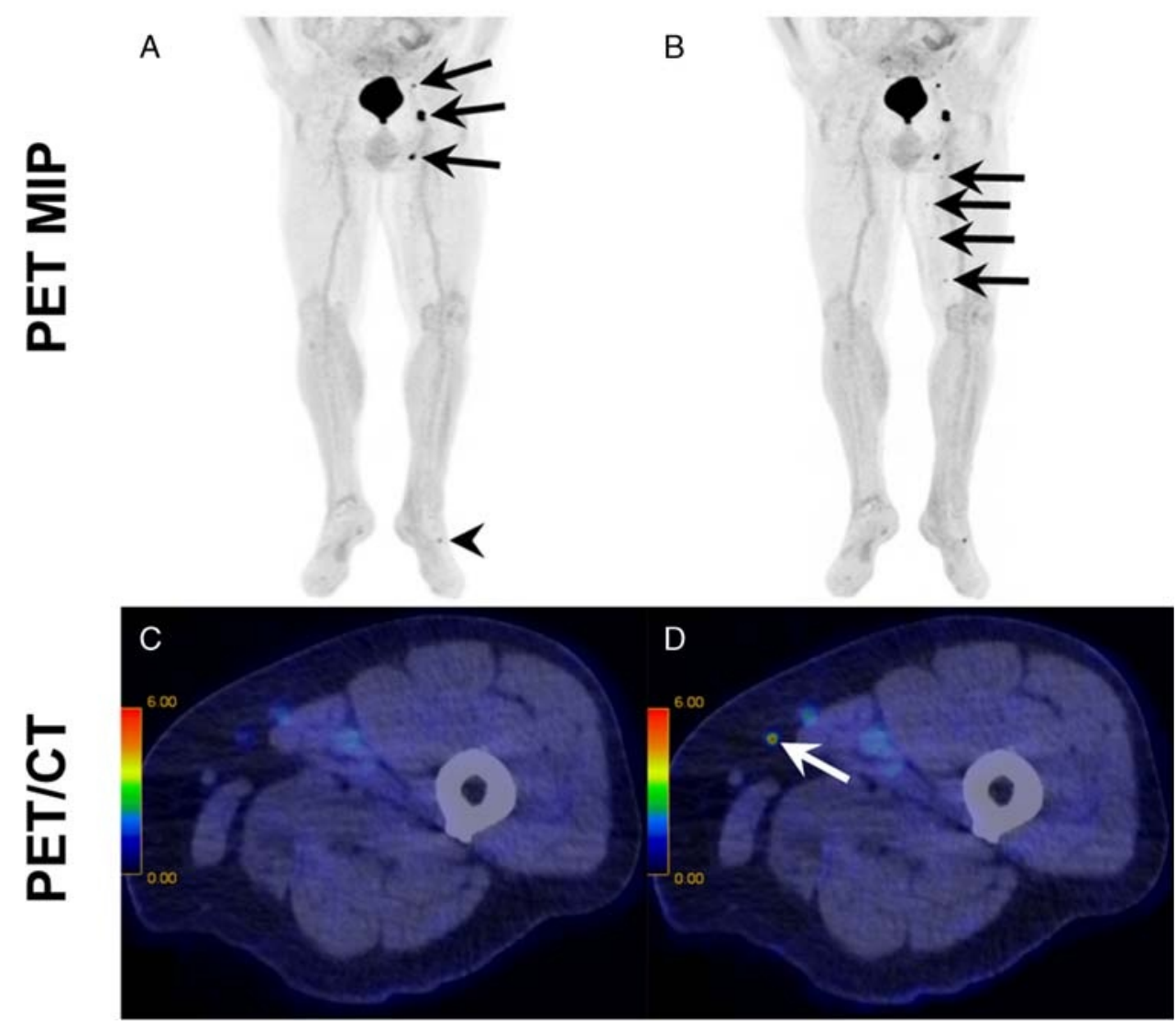

FIGURE 1. ${ }^{18}$ F-FDG-PET/CT imaging of a 70-year-old man with acral lentiginous melanoma, using a novel digital PET/CT system (Discovery MI PET/CT; GE Healthcare, Waukesha, WI). A dose of $267 \mathrm{MBq}$ of ${ }^{18} \mathrm{~F}-\mathrm{FDG}$ was administered. Coronal maximum intensity projection PET image reconstructed using ordered subset expectation maximization (OSEM) algorithm and point-spread function (PSF) (A) shows low uptake at the primary tumor excision site in the left foot (arrowhead). Several lymph node metastases (arrows) with high FDG uptake (SUVmax, 19.0) are seen in the inguinal and femoral region. On the identical dataset reconstructed with block sequential regularized expectation maximization (BSREM) (B), 4 additional FDG-avid foci (arrows) are easily discovered in the left thigh along the lymphatic stream, compatible with in-transit metastases, which was eventually proven by histopathology. In retrospect, some of them are also hardly seen on the OSEM + PSF reconstruction (A). Axial fused ${ }^{18}$ F-FDG-PET/CT image using OSEM + PSF reconstruction (C) at the level of one in-transit metastasis shows only very subtle uptake (SUVmax, 1.7) close to background activity in the subcutaneous adipose tissue medial to the sartorius muscle. The same lesion (arrow) is easily identified as distinct focal uptake (SUVmax, 6.1) on the axial fused ${ }^{18}$ F-FDG-PET/CT image using BSREM reconstruction, whereas the activity in a vessel nearby is not promoted (D). The American Joint Committee on Cancer (AJCC) defines in-transit metastases as any skin or subcutaneous metastases that are located more than $2 \mathrm{~cm}$ away from the primary lesion but are not beyond the regional nodal basin. They are considered intralymphatic extensions of the primary tumor before reaching the nearest lymph node. The incidence of in-transit metastases is $2 \%$ to $10 \%$ in melanoma patients, with $75 \%$ of these patients eventually developing either nodal metastases, systemic metastases, or both. ${ }^{1}{ }^{18} \mathrm{~F}-\mathrm{FDG}-\mathrm{PET} / \mathrm{CT}$ is widely used in the initial staging and therapy monitoring of malignant melanoma patients, particularly in advanced cases. ${ }^{2-5}$ Recently introduced digital PET detector technology yields higher intrinsic system sensitivity compared with the latest generation analog technology. ${ }^{6}$ PET reconstruction algorithms are known to have potential impact on staging in oncology patients. ${ }^{7-12}$ In our case, BSREM yielded a clinically relevant improvement over OSEM + PSF by detecting tiny malignant lesions. Block sequential regularized expectation maximization is a Bayesian penalized-likelihood reconstruction algorithm for PET, aiming to improve clinical image quality and allowing for absolute quantification. ${ }^{6,13-15}$ Block sequential regularized expectation maximization allows every single image voxel to reach full convergence. ${ }^{16}$ The penalty is designed such that edges are preserved while background image noise is kept low. ${ }^{16}$ Using an optimum penalized factor (beta) value of 450 as in our case yields a high contrast recovery and lower background variability in body FDG-PET images. ${ }^{6,11,15,17,18}$ The use of BSREM may have implications notably for the detection of small lesions, such as in-transit metastases as in our case or lymph node metastases. $6,10,11$ This is particularly important for malignant melanoma patients, whose prognosis worsens with the presence of in-transit metastases, and even more so if they are missed. 